\title{
PODERES LOCALES Y GLOBALES. UN ESTUDIO DESDE LAS EVALUACIONES DE IMPACTO AMBIENTAL EN LAS TONINAS, PARTIDO DE LA COSTA, BUENOS AIRES, ARGENTINA*
}

\author{
Eleonora Marta Verón ${ }^{1}$ y María del Carmen Villar ${ }^{2}$ \\ ${ }^{1}$ CONICET - Departamento de Geografía - Facultad de Humanidades - Universidad \\ Nacional de Mar del Plata - Funes 3350 nivel 2. Mar del Plata. Argentina \\ eleonorav@mdp.edu.ar. \\ ${ }^{2}$ Departamento de Geografía - Facultad de Humanidades - Universidad Nacional de \\ Mar del Plata - Funes 3350 nivel 2. Mar del Plata. Argentina \\ mcvillar@mdp.edu.ar.
}

\begin{abstract}
Resumen: Las nuevas tecnologías de la información y comunicación constituyen el principal soporte material en el proceso de globalización, siendo uno de los mayores adelantos la utilización de cables submarinos de fibra óptica. Por ello es que en el presente trabajo pretendemos abordar-desde una perspectiva de análisis geográfica -la temática de las telecomunicaciones a escala global y sus implicancias ambientales en el ámbito local. Nuestro propósito general es poner en evidencia, las relaciones de fuerza disímiles que se establecen entre el poder emanado de las empresas transnacionales con aquellos que puedan emerger en el ámbito local. Todo ello en el contexto de un país periférico, poniendo la mirada en el tratamiento de las cuestiones ambientales involucradas con la irrupción de proyectos de telecomunicaciones de escala global materializados al interior de la localidad atlántica de Las Toninas, Pdo. de La Costa, Provincia de Buenos Aires. El foco de esta mirada ambiental estará ceñido al análisis crítico de las Evaluaciones de Impacto Ambiental que emergen en ese proceso. En la indagación fueron utilizadas técnicas cualitativas multivariadas.
\end{abstract}

Palabras clave: Relaciones de poder - Evaluaciones de Impacto Ambiental Telecomunicaciones, Fibra Óptica - Las Toninas, Partido de la Costa, Argentina.

\begin{abstract}
Abstrac: The new technologies of information and communication are the main material in the process of globalization, one of the greatest advances in the use of fiber optic cables. That is why in this article tries to address, from a geographical analysis of the issue of telecommunications and global environmental implications at the local level. Our overall purpose is to highlight the power rela-
\end{abstract}

* Recibido: 24-04-09. Aceptado: 28-06-10. 
tions being established between dissimilar power emanating from transnational corporations with those that may emerge in the local area. All this in the context of a peripheral country, paying attention to the treatment of environmental issues involved with the explosion of telecommunications projects materialized global scale within the locality of The Atlantic Toninas, Pdo. La Costa, Buenos Aires Province. The environmental focus of this look is tight to the critical analysis of Environmental Impact Assessments that emerge in this process. In the qualitative inquiry were used multivariate techniques.

Keywords: Power relationship - Environmental Impact Assessments Telecommunications, Optic Fiber - Las Toninas, Partido de la Costa, Argentina.

\section{Introducción}

Desde la geografía se considera al espacio como un producto del accionar de las sociedades sobre la naturaleza con el fin de satisfacer sus necesidades. Por ello no puede pasarse por alto que el modelo de acumulación vigente caracterizado por ser expansionista y por su necesidad de aumentar su tasa de ganancia reproduciendo constantemente el capital. Para ello amplía las fronteras incorporando nuevos mercados, territorios e internacionalizando la producción. Ello ha llevado a hablar de globalización, proceso que intensifica las relaciones sociales a escala mundial, de modo que los vínculos creados eslabonan lugares distantes y hace que los sucesos locales se vean configurados de manera concreta por sucesos geográficamente remotos (HARVEY, 1995, 2001; CICALESE, 2000). SANTOS (2000) define su condición más importante como "perversa" porque las formas concretas dominantes de realización de la globalidad son el empobrecimiento material, cultural y moral, hechos posibles por el discurso y la práctica de la competitividad a todos los niveles. Los flujos de información -tal como se desarrollan en la actualidad- son identificados como el principal salto cualitativo de la nueva situación. (TABALES, 1997; MITTELMAN, 1996; HIRDS et al., 1999; HARVEY, 2005). En este sentido, y según lo expuesto por QUINTAR et al (2001), disminuye la importancia relativa de las distancias, y la cooperación productiva y la eficiencia dejan de depender de los grados de proximidad y de la centralización territorial.

En este marco, las nuevas tecnologías constituyen el principal soporte material para la integración de los procesos económicos a nivel mundial, al mismo tiempo que cobra mayor importancia el rol de las ciudades como soporte material de los procesos productivos estructurados e integrados globalmente. Unos de los mayores adelantos de las nuevas tecnologías de la información y la comunicación se encuentran en la utilización de cables submarinos de fibra óptica, que permiten transmitir una extraordinaria cantidad de información a grandes distancias, en tiempo real y con 
gran calidad. Y es dentro de este contexto que determinados territorios locales se han perfilado como espacios aptos para permitir esta transmisión. En ese momento las verticalidades del territorio se manifiestan y las empresas multinacionales actúan sobre el espacio local. A ello se suma que las empresas de telecomunicaciones al competir en el mercado global se realizan como tales mediante la búsqueda egoísta de alternativas que exclusivamente garanticen su sobrevivencia sin tomar en cuenta los efectos espaciales que puedan generar en los territorios sobre los que se instalan (SANTOS, 1999, HARVEY, 2001). Es por ello que resulta interesante tener en cuenta su comportamiento al analizar el territorio local.

Es a partir de estas consideraciones que en el presente trabajo se pretende abordar -desde una perspectiva de análisis geográfica- las relaciones de fuerza disímiles que se establecen entre el poder emanado de las empresas transnacionales con aquellos que puedan emerger en el ámbito local. Ello teniendo en cuenta el contexto de un país periférico, y poniendo la mirada en cómo se ha tratado la cuestión ambiental con la irrupción de proyectos de telecomunicaciones de escala global materializados al interior de la localidad atlántica de Las Toninas, Partido de La Costa, provincia de Buenos Aires. Todo con el fin de generar una red de comunicación subcontinental que consolide la malla comunicacional que liga los principales centros urbanos del orbe. El foco de esta mirada ambiental estará ceñido al análisis crítico de las Evaluaciones de Impacto Ambiental que emergen en ese proceso.

\subsection{Marco Metodológico utilizado}

En el método cualitativo de indagación fueron utilizadas técnicas cualitativas, habiendo recurrido básicamente, a la realización de entrevistas a informantes claves calificados y al análisis de normativas y documentos oficiales. En lo que respecta a las primeras se realizaron entrevistas focalizadas / en profundidad (TYLOR, et al, 1987), con una muestra intencional o selectiva previamente determinada en función del objeto del trabajo (CASTRO, et al, 1997; FLICK, 2004; COOK, et al, 2005). Los entrevistados seleccionados fueron: representantes del sector medioambiental en la Municipalidad de la Costa entre los años 1995-2001; representantes de Telefónica SAUNISUR y ATLÁNTIS II en Las Toninas entre los años 1994 a 2007; y, representantes de Telefónica SA-EMERGIA en Las Toninas entre los años 1994 a 2007. Las entrevistas fueron efectuadas con el objeto de comparar puntos de vista sobre el tema de los actores involucrados más importantes, para conocer aspectos centrales de las evaluaciones de impacto ambiental por sus propios elaboradores y evaluadores, y, para desentrañar a partir del análisis del discurso las relaciones de poder entre las empresas transnacionales - propietarias de los cables submarinos- y los gobiernos localesMunicipalidad de la Costa. 
Se analizaron diversos documentos bibliográficos que sirvieron para la elaboración de la base teórica. Se consultó la normativa vigente sobre cuestiones ambientales en general y evaluaciones de impacto ambiental en particular, tanto a escala nacional (LEY $\mathrm{n}^{\circ}$ 25.675/02), provincial (LEY $\mathrm{n}^{\circ}$ 11.723/95) y municipal (ORD MUN $\mathrm{n}^{\mathrm{o}}$ $1.542 / 95$, ), con el objeto de comparar aquello que en la norma rige para la instalación de este tipo de proyectos con lo que realmente ha sucedido. Para ello se analizaron los expedientes de las Declaraciones de Impacto Ambiental de los proyectos Atlantis II, SAC y SAM 1 (ORD MUN nº 2959/99, 2753/04, 2870/05; EXPEDIENTE MUNICIPAL 060/00).

Otros recurso que resultó de gran utilidad en el proceso analítico fue el reconocimiento de campo que permitió la contrastación entre la información obtenida vía entrevistas y análisis documental y la verdad terrena (ground true).

\subsection{Una aproximación conceptual}

Al campo de acción sociedad-naturaleza con sus diversidades se lo denomina ambiente. Cuando el hombre realiza sus actividades dentro de un modo de producción determinado genera ciertos efectos sobre la naturaleza. Los mismos pueden ser beneficiosos o nocivos, según el tipo de organización que adopten y según el peso que se le otorguen a las consideraciones temporales de la naturaleza o a las netamente humanas (estas últimas, por lo general, más en consonancia con las lógicas economicistas) respectivamente; vale decir, según su racionalidad productiva dominante. $\mathrm{Y}$ es en ese momento en que se van manifestando las diferencias en las racionalidades (natural y social) cuando se habla de problemas ambientales. Esto es, el exceso de carga de las demandas o consumo social sobre un soporte natural, o una incapacidad del soporte natural de reaccionar para disminuir la carga. El problema ambiental no puede entenderse como un efecto aislado de la relación sociedad-naturaleza, sino como resultante de largos y complejos procesos de antropización. Se manifiesta como tal, siempre y cuando exista un sujeto social que se vea afectado, quien variará según el contexto, es decir, no todos los sujetos percibirán igual una determinada situación problemática, ni se verán afectados de la misma manera.

En los últimos años y bajo el manto del paradigma del desarrollo sostenible, la gestión ambiental se encuentra en las agendas oficiales de todo el planeta a distintas escalas. Es definida como el conjunto de acciones encaminadas a lograr la máxima racionalidad en el proceso de decisión relativo a la conservación, defensa, protección y mejora del medio ambiente (ESTEVAN,1995). No obstante en aquellos países denominados en vías de desarrollo por el establishment (entre los que se encuentra Argentina) que han adoptado -o debido adoptar- modelos de despegue en consonancia con unas lógicas de mercado poco proclives al respeto por el ambiente y a las 
que poco le importan las consecuencias ni la generación de externalidades negativas que resulten de sus acciones, esta cuestión se hace más ardua. Claro está que el proceso de incorporación de las prácticas de gestión ambiental aún encuentra dificultades para infiltrarse contundentemente en las acciones de gestión en general o bien se encuentra meramente circunscripto al plano de las estrategias discursivas de los gobernantes de turno. Sin embargo, y a pesar de las formas lentas de penetración de las cuestiones ambientales en las esferas gubernamentales, los avances han sido significativos respecto a décadas anteriores, habiendo podido concretar ciertos instrumentos ad hoc que permitan aproximarse a un mejor manejo del ambiente .

Al respecto, y en lo que concierne a los aspectos legales inherentes al ámbito nacional, Argentina cuenta con la aprobación de una ley marco (LEY GENERAL DEL AMBIENTE, 2002) que entiende sobre la cuestión ambiental aunque la misma no esté aún reglamentada y, por tanto, se encuentre inhibida en su aplicación. Un análisis del articulado que la conforma permite reconocer que dicha ley prevé -en el artículo ${ }^{\circ}$ 8- la creación de los principales instrumentos de la política y la gestión ambiental nacional, entre los cuales se encuentra la evaluación de impacto ambiental. Y más específicamente, en su artículo $\mathrm{n}^{\circ} 11$ expresa que: "toda obra o actividad que, en el territorio de la Nación, sea susceptible de degradar el ambiente, alguno de sus componentes, o afectar la calidad de vida de la población, en forma significativa, estará sujeta a un procedimiento de evaluación de impacto ambiental, previo a su ejecución”. La evaluación de impacto ambiental es el conjunto de estudios y sistemas técnicos que permiten estimar los efectos que la ejecución de determinado proyecto, obra, o actividad causa sobre el ambiente en todas sus etapas (WATHERN, 1998, MORRIS et al, 2001). Es un procedimiento, instrumento de gestión ambiental que asegura un proceso de toma de decisiones adecuado al interés público. Dentro de ese proceso las autoridades competentes, deben realizar una Declaración de Impacto Ambiental, un documento público mediante el cual se revisan procedimientos y resultados, y se aprueba, condiciona o rechaza el proyecto (ECHECHURRI et al, 2002, PETTS, 1999).

Según PEREYRA et al (2001), en Argentina puede visualizarse una carencia de un marco legal integral de evaluaciones de impacto ambiental que armonice los procedimientos. Ello porque desde lo legal es usual que las legislaciones ambientales generales o de evaluaciones se centren en los aspectos físicos del ambiente especificando apartados tales como "del suelo", "de la atmósfera", "de las aguas", "de la flora", "de la fauna" etc, sin tener la misma consideración o minimizando "las cuestiones sociales". Y en tal sentido, las Declaraciones de Impacto Ambiental que se desprenden de las evaluaciones de impacto ambiental a analizar en los siguientes apartados (ORD MUN no 2959/99, 2753/04, 2870/05) y que hacen referencia a los emprendimientos realizados por las grandes empresas en el distrito de La Costa, Buenos Aires, Argentina, no están exentas de estas características, a las que se le adi- 
cionarán otras particularidades que las hacen por demás interesantes desde el punto de vista analítico.

\section{Estudio de caso}

\subsection{Llegada de redes de telecomunicación en el litoral marítimo argentino}

El área de estudio es una pequeña localidad del litoral marítimo bonaerense llamada Las Toninas, en el Partido de la Costa, provincia de Buenos Aires, Argentina, lugar en el cual se materializan las principales obras ingenieriles que hacen posible uno de los mayores adelantos de las denominadas nuevas tecnologías de las telecomunicaciones (Fig. 1).

La población estable del distrito según el último Censo Nacional de Población y Vivienda es de 60.483 habitantes, de los cuales 3.550 residen en Las Toninas (INDEC, 2001). La principal actividad económica de la localidad, al igual que la del partido en la que se inserta, es el turismo. Éste se manifiesta como motor del desarrollo, incentivando y promoviendo un conjunto de actividades asociadas, constituyéndose en generadora de empleo. El turismo desarrollado en el área es el llamado turismo de masas y sol y playas (BERTONCELLO, 1993), que se basa en el disfrute de características paisajisticas y recreativas del entorno natural que se valorizan, como la temperatura del ambiente, del agua, el sol, la arena, entre otras.

A partir de los años 90 del siglo XX debido a los nuevos requerimientos mundiales sobre el aumento de las capacidades de transmisión de datos e información en materia de comunicaciones (Internet, comercio electrónico, videoconferencia, tele presencia), se impuso el desarrollo masivo de nuevas formas de conducir la información a través de cableados submarinos de fibra óptica, por intermedio de grandes empresas que realizarán considerables inversiones en el sector. Las fibras ópticas son conductos de fibra de vidrio que se utilizan para transmitir todo tipo de información previamente digitalizada en forma de ases de luz a gran velocidad, en tiempo real, y con gran calidad. Son recubiertas por un cable -cable submarino de fibra ópticaencargado de contener y proteger las fibras, además de otras funciones técnicas. El mismo, se instala bajo el agua, enterrado en las plataformas cercanas a las costas, y apoyado sobre el lecho marino en altamar. Al ingresar al continente los cables necesitan de "puertos donde amarrar", las Estaciones de Amarre, edificaciones que alojan los equipos que deben instalarse en cada extremo del cable (equipos terminales), que reciben, alojan y reorientan la información, y aquellos necesarios para controlar el cable. 


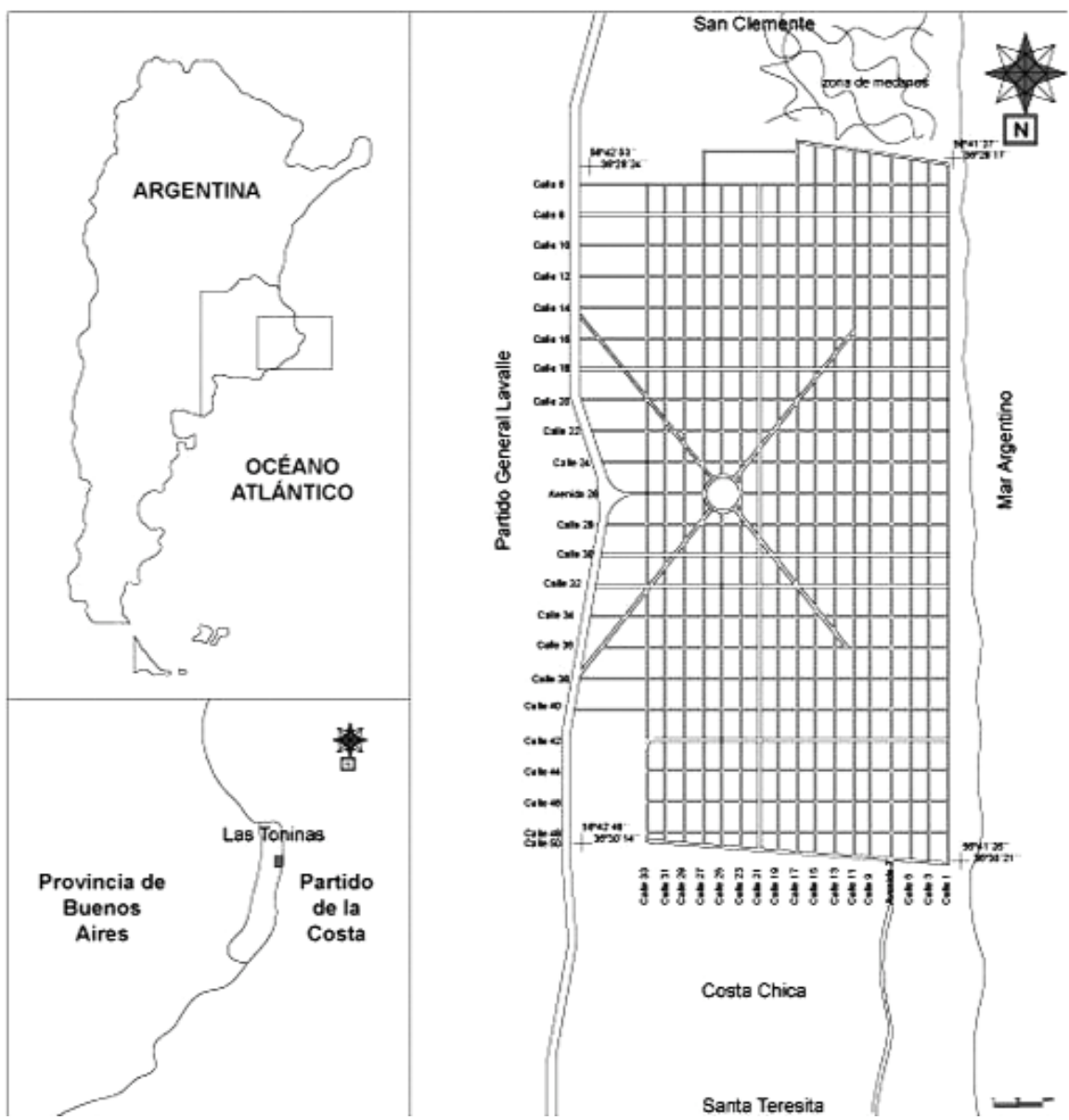

Figura 1. Situación del área de estudio.

En Argentina estas inversiones comenzaron a materializarse a partir de mediados de los años 90 del siglo pasado, momento en el que arribó al territorio nacional el primer sistema de cable submarino, denominado Unisur. A éste le seguieron otros tres (Atlantis II, Sac y Sam 1), que rápidamente se fueron modernizando, aumentando su capacidad y velocidad de transmisión y en algunos casos variando sus recorridos para unir las costas latinoamericanas y europeas. La primera de las redes submarinas que arribó al país es la denominada Unisur, en el año 1994, también llamado "El cable del Mercosur", uniendo Argentina (Las Toninas), Uruguay y Brasil a lo largo de la costa atlántica. La segunda red, y quizás la más importante teniendo en cuenta su recorrido, 
es Atlantis II, que se materializó en el año 1999 conectando Argentina (Las Toninas), Uruguay, Brasil, Senegal, Is. Cabo Verde, España y Portugal. El principal inversor tanto de Atlantis II como de Unisur fue el Grupo Telefónica SA (TELEFÓNICA, 1997). En el año 2001, arribó al país el sistema SAM 1 que conecta Argentina (Las Toninas), Brasil, Puerto Rico, Estados Unidos, México, Guatemala, Colombia, Ecuador, Perú, y Chile, siendo el sistema de mayor capacidad de transmisión de América Latina, propiedad de la empresa Emergia, subsidiaria de telefónica de Argentina (EMERGIA, 2001). Finalmente, el último sistema en llegar a la Argentina, fue el denominado SAC, también en el año 2001, con un recorrido similar al anterior sistema, pero propiedad de la empresa Global Crossing.

Todos los cables submarinos de fibra óptica tuvieron como destino y punto de convergencia territorial la localidad de Las Toninas, lugar seleccionado como sitio ideal dadas sus excelentes condiciones de accesibilidad a los grandes centros urbanos del país y a las características topográficas favorables de su lecho marino y playas. Vale la pena destacar que estos cables atraviesan los océanos siguiendo unas trayectorias precisas que sólo son conocidas por los navegantes que se valen de cartas náuticas actualizadas, monitoreadas celosamente por las grandes empresas inversoras por razones específicas de seguridad y que, al llegar a tierra firme, posibilitan la unión del país receptor con otros.

\subsection{Normativa y actores involucrados}

Pero ¿qué tipo de leyes y actores intervinientes implica la llegada de estos sistemas de telecomunicaciones a Argentina? En primer lugar, los marcos legales internacionales autorizan el despliegue de redes en la medida que las áreas oceánicas y sus fondos asociados, en la llamada alta mar, son considerados res comunnis (una cosa afectada al uso de todos). El artículo 112 de la CONVEMAR (1982) -Convención de las Naciones Unidas sobre Derecho del Mar, madre de las normativas marítimas internacionales- especifica que todos los estados tienen derecho a tender cables y tuberías submarinas en el lecho de la alta mar. De la misma manera, dicha normativa avala el derecho de los estados a tender cables submarinos por la plataforma continental y la zona económica exclusiva, aclarando que el estado ribereño, a reserva de su derecho a tomar medidas razonables para el control de la contaminación causada por cables, no puede impedir el tendido o la conservación de los mismos (ART n 79).

En lo que respecta al ingreso de los cables en el Mar Argentino, la esfera nacional debe autorizar el acceso de los mismos - previo permiso de paso- sobre los fondos marinos que se asocian al mar territorial donde tiene soberanía absoluta, ya que dicho paso es considerado por la CONVEMAR como inofensivo. Y por ultimo, al arribar al sector playas los cables submarinos compromete la actuación de los sectores provincial y municipal. 
En cuanto al tipo de obras que se requirieron para concretar estas instalaciones, se hizo necesario construir en tierra firme estaciones de recepción y trasvase de información y realizar tareas que permitieran la llegada segura y eficiente del sistema de redes (tendido del cable, enterramiento, instalación de tanquilla de empalme y banco de electrodos, etc.). Estas tareas comprometieron a espacios públicos y privados cercanos e inmediatos al área de playas que, en el caso de la localidad argentina de Las Toninas, como ya se comentara, constituyen su mejor recurso y su mayor atracción y fuente de ingresos dado su orientación monoproductiva hacia el turismo de sol y playas. Se realizaron zanjeos para enterrar el cable hasta la primer calle después del médano donde se instalaron las llamadas tanquillas de empalme o banco de electrodos (la puesta a tierra del cable para que cierre el circuito eléctrico que alimenta los repetidores con el mar, a una profundidad de $10 \mathrm{~m}$..) y, cuando los cables ingresaron dentro de la trama urbana, lo hicieron vía subterránea, para luego distribuirse a lo largo del país.

Vale aclarar que cuando estos sistemas arribaron a esta localidad estuvieron acompañados de discursos oficiales y oficiosos que prometían insertar a la localidad en el mundo, otorgar mayores fuentes de empleos a sus habitantes y mejorar las condiciones de vida generales de la región (PIONERO, 1993). Contrariamente a lo esperado no sólo no se generaron más empleos, ni se mejoraron las condiciones de vida generales de la población involucrada, sino que, paradójicamente, tampoco mejoraron las comunicaciones en el área, ya que, la localidad de Las Toninas aún no ha podido beneficiarse de todos los servicios que brindan estos sistemas (VERÓN, 2004).

Desde el punto de vista ambiental y dado que la red atravesó espacios de distinta jurisdicción y competencia, se hizo necesaria la intervención de diferentes autoridades que, en el caso de las obras emprendidas por las empresas en Las Toninas, involucraron a la entonces Secretaría de Política Ambiental de la provincia de Buenos Aires (hoy Organismo Provincial para el Desarrollo Sustentable) y a la Municipalidad de La Costa, quienes debieron controlar y supervisar dichas obras, según la normativa vigente. Este procedimiento se encontró enmarcado dentro de la ley provincial $\mathrm{n}^{\circ} 11.723 / 95$ (que entiende y regula los aspectos ambientales bonaerenses) y la ordenanza municipal 1.542/95 que establece, en su artículo $n^{\circ} 1$, que todo proyecto público o privado, consistente en la realización de nuevas obras o instalaciones o en la prestación de servicios que pudiesen afectar el ecosistema costero deberán contar con una evaluación de impacto ambiental previa y la declaración de impacto ambiental.

A esta altura de las formulaciones precedentes, y en un contexto espacio-temporal en que el estado nacional, en general, ha apostado y apuesta por un proceso de descentralización como una forma de abandono de algunas de sus funciones otrora indelegables, cabe preguntarse ¿con qué instrumentos y con qué poder podría el dis- 
trito de La Costa, de 60.000 habitantes, enfrentar a los grandes grupos económicos en esta relación entre poderes globales y locales? ¿con qué fuerzas podría desafiar esta situación que pone en riesgo potencial a sus recursos ambientales más preciados? La alternativa municipal estuvo encaminada a formular un marco legal acorde y una reestructuración institucional que pudiera, al menos, ejercer un determinado control y presión ante las acciones ejecutadas por las empresas en su propio territorio. En efecto, la aprobación de la ordenanza municipal $n^{\circ} 1.542 / 95$ y la creación del Departamento de Costas y Medio Ambiente emergieron en sincronía con la llegada de los emprendimientos en cuestión, en un intento de protección ambiental local. Como lo expresara en la entrevista realizada un empleado de una de las empresas propietarias de los cables “... en el tema ambiental se mueven muchos intereses personales. Cuando se bizo, cuando se trajo el Unisur en el año 1994 la municipalidad no estaba preparada, no tenía estructura ni nada para capitalizar de alguna manera la entrada del cable. Pero después como vino el Unisur, armaron una estructura.." Y fue a partir de la obligación de realizar las evaluaciones de impacto que estas grandes compañías tuvieron que relacionarse -dando cuenta de sus acciones- de una forma más directa con el municipio. Pero ¿Cómo fueron desarrollándose estas relaciones basándose en los requerimientos planteados por el gobierno local? En las próximas líneas se intentará dar cuenta de ello.

\subsection{Relación entre poderes locales y globales en torno a las Evaluaciones de Impacto Ambiental}

De los cuatro sistemas que arribaron al país, sólo tres de ellos (Atlantis II, Sac y Sam1) contaron con evaluación de impacto ambiental, y, por lo tanto, con Declaraciones de Impacto Ambiental (ORD MUN no 2959/99, 2753/04, 2870/05). Unisur no realizó ningún tipo de evaluación argumentando que la normativa municipal que obliga a la aplicación de este tipo de instrumento es del año 1995 y las obras del sistema en cuestión habían comenzado a desarrollarse en el año anterior.

Puede afirmarse que, en todos los casos, la relación municipalidad-empresa en lo que respecta a la realización de las evaluaciones fue "conflictiva", ya que fue necesario hacer de forma oficial una serie de notificaciones a las multinacionales (envíos de cartas, documentos, y otras medidas intimidatorias semejantes - EXPEDIENTE MUNICIPAL 060/00) para que dichas evaluaciones fueran ejecutadas. No obstante ello, y una vez concretadas, se visualiza que o bien fueron confeccionadas por consultoras que contrataron las mismas empresas, con lo cual se pone en duda que el contratado incorpore en sus informes efectos negativos que puedan perjudicar los intereses económicos de quienes lo contratan, es decir, la "patronal"; o bien los encargados de llevarla a cabo estuvieron íntimamente relacionados (relaciones de intereses y/o de subordinación política) con quienes tomaron la decisión de aprobarlas o desaprobarlas. 
Asimismo, una vez analizados las Declaraciones de Impacto Ambiental (ORD MUN no 2959/99, 2753/04, 2870/05) que correspondes a las evacuaciones de impacto ambiental pueden realizarse algunas apreciaciones generales de los mismos. Las empresas argumentaron que sus obras no causarían grandes impactos porque las mismas seguirían las mismas rutas y procedimientos que el proyecto Unisur ya instalado $\mathrm{y}$ al que presentaban como un antecedente, antecedente no válido si se recuerda que para dicho emprendimiento no se presentó ninguna documentación. De la misma manera, dichas evaluaciones obviaron los aspectos sociales, teniendo como centro de atención exclusiva las tareas de enterramiento de cables en playas y zonas de médanos. En la etapa de construcción las evaluaciones no encontraron impactos ambientales importantes porque el área se encuentra bajo un proceso de erosión severo; no obstante, mencionaron como los principales, el rebajamiento de los médanos en la zona afectada, la remoción de arena, y la impermeabilización del suelo por la construcción de la estación. En cuanto a los impactos en la etapa operativa, enunciaron como primordial el generado en la playa cuando el cable quede al descubierto durante su vida útil (25 años), después de algún evento extraordinario o por efecto de la importante erosión costera en el área, para lo cual se previó un plan de monitoreo permanente de la zona de playa y médanos. Las Declaraciones de Impacto Ambiental agregaron a ello la alteración sobre la fauna, la flora y plancton del lugar y la afectación de la calidad de las aguas costeras por posible contaminación. Asimismo, se estableció que las empresas debían enterrar el cable en zonas donde había peligro de enganches con redes de pesca, alertar a los usuarios marinos de su existencia, elaborar mapas con la ruta del tendido para entregárselos a los organismos interesados y realizar medidas de señalización adecuadas. Además, debían entregar al Departamento de Costas - cada seis meses- perfiles de playa en los que se indique la trayectoria del cable y las variaciones de las costas y elevar un informe del estado de los componentes físicos, biológicos y afines a la Municipalidad de la Costa cada 12 meses por un periodo de 20 años.

En primer término, y haciendo referencia a los impactos y mitigaciones detalladas para el caso que el cable quede al descubierto, vale la pena revisar algunas cuestiones. Los cables tienen una vida útil de 25 años y los acuerdos de monitoreo y control del cable entre la Municipalidad de la Costa y las empresas son por 20-25 años. A ello se le suma que no aparece claro en ninguna documentación qué es lo que van a hacer las empresas pasado ese tiempo, sólo se menciona que llegado el momento se evaluará el tema. Ante ello cabe preguntarse ¿qué pasara con el cable una vez concluido dicho período? ¿quién se hará cargo del mismo?. Cabe pensar quién lo desenterrará si esos son los planes, o monitoreará para evitar que quede expuesto a la superficie si no se planea extraerlo. Este es un aspecto central para replantearse, dado que es importante en todo estudio de impacto ambiental incluir los impactos y medidas de mitigación para la etapa de abandono del proyecto, que suele ser la más descuidada y, en muchas ocasiones, la más impactante. 
De las medidas de mitigación recomendadas a las empresas, pocas han sido las que efectivamente se han cumplido en tiempo y forma. En lo que concierne a las entregas de los perfiles de playa y de informes en los que se detalle el estado de las mismas cada seis meses ante la municipalidad, las empresas no las realizaron ni las realizan a término y no se ha llevado a cabo ninguna reunión entre técnicos de ambas partes para evaluar la evolución de los efectos de la dinámica costera y expresar un diagnóstico (Entrevista a funcionario de la Municipalidad de la Costa entre los años 1993-2001). A ello se le suma una serie de irregularidades en los gráficos como no especificar el recorrido del cable, ausencia de referencia alguna ni escala, con lo cual resulta imposible realizar monitoreos de los posibles impactos por remoción de fondos (En EXPEDIENTE MUNICIPAL 060/00). Como complemento se debe remarcar que dentro del ámbito municipal y de los sectores encargados de la sección medioambiental, en el momento de las entrevistas realizadas se encontraron ciertas reticencias para hablar sobre la temática específica y sobre las empresas en general... ¿toma de conciencia de los poderes diferenciales involucrados en la relación?... Sin embargo, las entrevistas con empleados y ex empleados de las compañías fueron más abiertas y, en líneas generales, la mayor cantidad de las mismas fueron proclives a defender las posturas de los actores hegemónicos y sus acciones. Y en sus respuestas puede advertirse la conflictividad en las relaciones con el estado, ya que como expresara un empleado "...en el Depto. de Costas y Medio Ambiente de la Municipalidad de la Costa se enojaron. Un día pasó una inspección y vieron que se habia movido medio metro cúbico de arena y nos bicieron una multa de 45 mil mangos, pero eso es parte del gobierno que quiere sacar su tajada, obvio. O sea, una empresa que está trayendo cables en el año 2001, con 400 millones de dólares, puede pagar una sanción..."

\section{Conclusiones}

Las verticalidades del espacio se han manifestado en el espacio costero bonaerense, verticalidades que dejan ver la perversidad del proceso globalizador, gracias al cual las relaciones de poder diferencial se hacen evidentes y generan que los actores hegemónicos pongan las reglas del juego, y aquellos que no tienen el poder suficiente, como los gobiernos locales, deban acatar de la mejor manera las decisiones de los primeros. Sobre todo en auge descentralizador donde el estado nacional cada vez delega más responsabilidades pero menos recursos para enfrentarlas a los gobiernos locales. Y los marcos normativos, tanto internacionales como nacionales, favorecieron los intereses económicos de los agentes hegemónicos. Es innegable la distancia substancial que existe entre centro y periferia en lo que respecta a la disponibilidad de recursos tecnológicos. 
Por otra parte y, a escala nacional ¿es fortuito que la Ley General del Ambiente no esté aún reglamentada? ¿o es una mera dilación que le permite -a esta instancia del estado- relegar algunas de sus obligaciones primarias delegándolas a las impotentes administraciones locales? Ante esta situación, ¿con qué otro tipo de instrumentos que no sean los ambientales podría hacer frente un pequeño municipio a las grandes empresas transnacionales que tienen un respaldo de los principales poderes políticos de orden lógico superior?. Las únicas herramientas que dispone el ámbito municipal para trasformarse en un interlocutor válido que pueda enfrentar a los grandes poderes económicos en pos de la protección de su ambiente han estado circunscriptas a la reforma institucional (creación de una unidad específica de inspección y control) y a las aristas medioambientales de ciertos marcos normativos locales.

De las Declaraciones de Impacto Ambiental analizadas, documento final de las evaluaciones de impacto ambiental realizadas se desprende que están en consonancia con el espíritu general que, hasta el presente, ha tenido la tendencia en la realización de evaluaciones en el país, es decir: se han replicado las técnicas y procedimientos sin tener en cuenta las particularidades del área donde se aplican (en este caso, por poner solo un ejemplo, no se ha internalizado el concepto de litoral como un sistema complejo). Además el concepto ambiente ha sido restringido a los componentes naturales y biofísicos, obviando los sociales. Y por último son cuestionables tanto en los procedimientos utilizados como en el cumplimiento de los plazos y formas pautadas para la entrega de los informes de actuación y monitoreo. No obstante, si bien las evaluaciones que se realizaron a partir de los proyectos materializados pueden ser cuestionadas, en general han sido un instrumento válido para que los gobiernos locales como la Municipalidad de la Costa puedan ejercer cierto grado de presión, o control sobre estos grandes emprendimientos.

En la relación entre poderes globales y locales, por un lado está un pequeño distrito costero, y por el otro un conjunto de empresas multinacionales, empresas poderosas que no se preocupan por el ambiente en general, y menos aún cuando, la parcela ambiental en consideración constituye un territorio apropiado de un país periférico. Empresas poderosas cuya racionalidad es instrumental, cuyo espíritu es rentístico y sus horizontes inmediatos se sitúan en el corto plazo, siguiendo a la lógica productiva dominante. El accionar esquivo del cumplimiento de las normas pautadas por el Estado (un Estado a todas luces débil y subordinado), resultan de gran utilidad para estas empresas a quienes poco le importan los perjuicios que su accionar pueda ocasionar en el ambiente (natural y social) donde desarrollan sus prácticas. Ellas obtendrán ganancias inmediatas, y el costo socioambiental que el deterioro demande en el mediano y largo plazo, deberá ser afrontado por los espacios locales como externalidades económicas y políticas. Así, los pobladores de la localidad de Las Toninas, deberán soportar -durante gran cantidad de años- los cableados submarinos degradándose sobre sus playas, el principal recurso ambiental que -hasta el pre- 
sente- sostiene a su ya precaria situación socioeconómica. El estado debiera poder capitalizar el hecho de poseer el lugar ideal para la materialización de la globalización, y así desdibujar, al menos un poco, su perversidad.

\section{Bibliografía}

BERTONCELLO, R. (1993). "Configuración socio-espacial de los balnearios del Partido de la Costa (provincia de Buenos Aires)". Buenos Aires. Instituto Geografía, UBA. Pp. 190.

CASTRO, B y RODRIGUEZ, P (2005). "Más allá del dilema de los métodos: la investigación en ciencias sociales" Ed Norma. ISBN: 9580485429 . Pp. 421.

CICALESE, G (2000). "Los nuevos croquis territoriales de la globalización". En programa de capacitación para docentes del 3 o ciclo de EGB. Área Ciencias Sociales. Mar del Plata. UNMP. Pp. 23.

COOK, T Y REICHARDT, S (2005). "Métodos cualitativos y cuantitativos en investigación evaluativa" Ed. Morata. ISBN: 847112310X. Pp. 228.

ECHECHURRI H. Y FERRARO R. (2002). "La Evaluación de Impacto Ambiental". Mar del Plata. CIAM. UNMP. Pp. 530.

EMERGIA (2001). "Emergia finaliza el primer anillo de alta capacidad que une Latinoamérica y los Estados Unidos". www.emergia.com. [Consulta 12/12/2008]

ESTEVAN, A. (1995). "La monetarización del medio Ambiente y ecologismo de mercado. De la economía a la ecología”. 11, pp. 6778.

FLICK, U (2004). "Introducción a la investigación cualitativa" Ed Morata. ISBN: 8471124807. Pp. 322.

HARVEY, D (1995). "Globalization in Question" A Journal of Economics, Culture E Society, Vol 8, Issue 4, 1, pp. 1-17.
HARVEY, D (2001). "Spaces of capital: towards a critical geography" Ed Taylor \& Francis. ISBN: 0415932416. Pp. 492

HARVEY, D (2005). "Spaces of neoliberalization: towards a theory of uneven geographical development" Ed Franz Steiner Verlag. ISBN: 351508746X. Pp. 132.

HIRST, $P$ and THOMPSON, G (1991). "Globalization in question: the international economy and the possibilities of governance" Ed Wiley-Blackwell. ISBN: 0745621643. Pp. 318.

HONORABLE CONCEJO DELIBERANTE. MUNICIPALIDAD DE LA COSTA "Ordenanza Municipal $n^{-}$1542/95, 2959/99, 060/00, 2753/04, 2870/05".Mar del Tuyú.

INSTITUTO NACIONAL DE ESTADÍSTICA Y CENSO - INDEC (2001). "Censo Nacional de Población y Vivienda. Resultados Generales. Provincia de Buenos Aires. Localidades y Partidos de Argentina". CD 1. Buenos Aires.

MITTELMAN, J (1996). "Globalization: Critical Reflections" Ed JM. ISBN: 978-1-55587-7521. Pp. 223.

MORRIS, P AND THERIVEL, R (2001). "Methods of environmental impact assessment. The natural and built environment series" Ed Taylor \& Francis. ISBN: 0415239591. Pp. 492.

NACIONES UNIDAS (1982). "Convención de las Naciones Unidas sobre el derecho del Mar". 182 $2^{-}$sesión plenaria de la III Conferencia de las Naciones Unidas sobre 
el Derecho del Mar. Montego Bay (Jamaica)

PEREYRA, A. Y SORIA, M. (2001). "La dimensión social de la evaluación de impacto ambiental. De la Teoría a la Realidad". Tercer Encuentro Internacional Humboldt Salta, Argentina. Pp. 16

PETTS, J (1999). "Handbook of Environmental Impact Assessment" Hardcover. Vol I ISBN: 978-0-632-04772-7. Pp. 496.

PIONERO (1993). "Fibra Óptica en Las Toninas". Ed Pionero. Mar del Tuyú.

QUINTAR, A. Y VIO, M. (2001). "Sociedad Informacional y Nuevas Tecnologias Urbanas. Entre La Competencia y La Cooperación”. Eure v.27, n.1, pp. 101-113.

SANTOS, M. (2000). "Los espacios de la globalización". Documento en formato electrónico. [Consulta 12/12/2008]. http://www. ucm.es/BUCM/revistas/ghi/2119803/articulos/AGUC9393110069A.

SANTOS, M (1999). "Globalización y territorios: de la compartimentación a la fragmentación". En Velásquez y otros, Calidad de Vida Urbana, aportes para su estudio en Latinoamérica. Tandil. UNICEN. Pp. 15.

SENADO Y CÁMARA DE DIPUTADOS DE LA NACIÓN ARGENTINA (2002). "Ley $n^{-}$
25.675. Ley General del Ambiente". Buenos Aires.

SENADO Y LA CÁMARA DE DIPUTADOS DE LA PROVINCIA DE BUENOS AIRES (1995). "Ley no 11723/95". La Plata.

TABALES, A. (1997) "Neoliberalismo y territorio. Posibilidades de una nueva política regional en América Latina". Univ. Internac. de Andalucía. Ed del autor.

TELEFÓNICA SA (1997). "El grupo Telefónica participará en la construcción y mantenimiento del cable submarino Atlantis 2". Madrid.

TYLOR, S y BOGDAN, R (1987) "Introducción a los métodos cualitativos de investigación la búsqueda de significados" Ed Paidós. ISBN8475098169. Pp. 344.

VERÓN E. (2004) "La Estación de Amarre de Fibra Óptica en la localidad de Las Toninas, Partido de la Costa. Representaciones Sociales y Problemáticas Socioambientales". VI Jornadas de Investigación del Departamento de Geografía". Mar del Plata. Facultad de Humanidades. Departamento de Geografía. UNMP. Pp. 10

WATHERN, P (1998) "Environmental impact assessment: theory and practice" $\mathrm{Ed}$ Routledge. ISBN0415078849. Pp. 352. 